
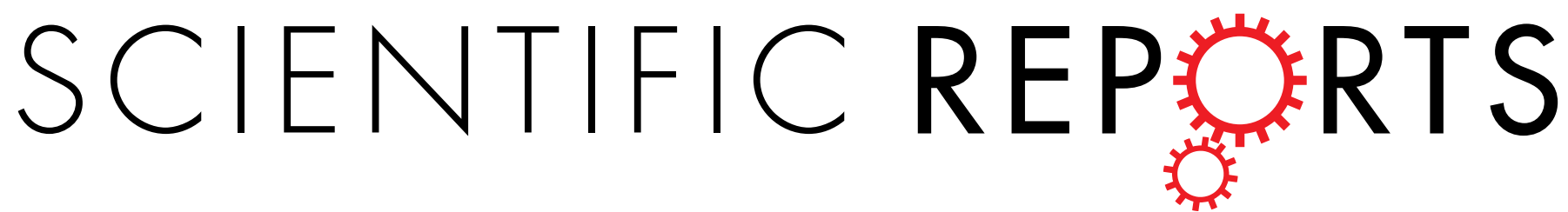

\title{
OPEN Global selection of Plasmodium falciparum virulence antigen expression by host antibodies
}

Received: 05 June 2015

Accepted: 21 December 2015

Published: 25 January 2016

\author{
Abdirahman I. Abdi ${ }^{1,2}$, George M. Warimwe ${ }^{4,5}$, Michelle K. Muthui ${ }^{1}$, Cheryl A. Kivisi ${ }^{1}$, \\ Esther W. Kiragu ${ }^{1}$, Gregory W. Fegan ${ }^{1,3}$ \& Peter C. Bull ${ }^{1,3,+}$
}

Parasite proteins called PfEMP1 that are inserted on the surface of infected erythrocytes, play a key role in the severe pathology associated with infection by the Plasmodium falciparum malaria parasite. These proteins mediate binding of infected cells to the endothelial lining of blood vessels as a strategy to avoid clearance by the spleen and are major targets of naturally acquired immunity. PfEMP1 is encoded by a large multi-gene family called var. Mutually-exclusive transcriptional switching between var genes allows parasites to escape host antibodies. This study examined in detail the patterns of expression of var in a well-characterized sample of parasites from Kenyan Children. Instead of observing clear inverse relationships between the expression of broad sub-classes of PfEMP1, we found that expression of different PfEMP1 groups vary relatively independently. Parasite adaptation to host antibodies also appears to involve a general reduction in detectable var gene expression. We suggest that parasites switch both between different PfEMP1 variants and between high and low expression states. Such a strategy could provide a means of avoiding immunological detection and promoting survival under high levels of host immunity.

Plasmodium falciparum erythrocyte membrane protein 1 (PfEMP1) is a diverse family of proteins that are inserted into the surface of $P$. falciparum infected erythrocytes (IE). PfEMP1 plays an important role in malaria pathology by mediating cytoadhesion of parasite IE to various host receptors including CD $36^{1,2}$, Intercellular adhesion molecule-1(ICAM-1 $\left.{ }^{3}\right)$, endothelial protein $\mathrm{C}$ receptor $(\mathrm{EPCR})^{4}$ on the vascular endothelial cells and complement receptor $(\mathrm{CR} 1)^{5,6}$ on erythrocytes ${ }^{7,8}$. Cytoadhesion of IE in the deep microvasculature allows parasites to avoid passage through the spleen where they would normally be removed from circulation ${ }^{9}$. When the parasite burden is high, this parasite survival strategy causes vascular occlusion contributing to the impaired perfusion thought to be the main cause of the distinct pathology associated with infections by this species of malaria parasite ${ }^{10}$.

PfEMP1 is encoded by a multi-gene family called $\operatorname{var}^{11-13}$. Each parasite genome contains approximately 60 var genes, which are expressed in a mutually exclusive manner ${ }^{14}$. Switches in var gene expression allow the parasite to evade host immunity and prolong infection by evading antibody response ${ }^{13,15,16}$. Parasites that survive within the host are those that express PfEMP1 variants corresponding to gaps in the endogenous repertoire of host antibodies ${ }^{17,18}$.

Despite their immense molecular diversity, mainly generated through recombination events ${ }^{19-24}$, var genes can be classified into three major groups, $\mathrm{A}, \mathrm{B}$, and $\mathrm{C}$ according to sequence features found in their $5^{\prime}$ un-translated region $^{14,25}$. These groups are broadly associated with the structural organization and size of the protein, with group A PfEMP1 tending to be longer than non-group A PfEMP1 ${ }^{26}$. Recently a functional classification based on the presence of commonly occurring combinations of specific Duffy binding-like (DBL) and cysteine-rich inter-domain region (CIDR) domains called domain cassettes (DCs) was described ${ }^{27}$.

Epidemiological data suggest that the risk of severe disease declines more rapidly than mild malaria as children grow older ${ }^{28,29}$. This more rapid acquisition of immunity to severe malaria as compared to mild malaria has

${ }^{1}$ KEMRI-Wellcome Trust Research Programme, P.O. Box 230-80108, Kilifi, Kenya. ${ }^{2}$ Department of Biochemistry and Chemistry, Pwani University, P.O. Box 195, 80108, Kilifi, Kenya. ${ }^{3}$ Nuffield Department of Clinical Medicine, John Radcliffe Hospital, University of Oxford, Oxford, OX3. ${ }^{4}$ The Jenner Institute, University of Oxford, ORCRB, Roosevelt Drive, Oxford, OX3 7DQ, UK. ${ }^{5}$ Centre for Research in Therapeutic Sciences and, Institute for Healthcare Management, Strathmore University, P.O. Box 59857-00200 Nairobi, Kenya. ${ }^{\dagger}$ Present address: Department of Pathology, University of Cambridge, Tennis Court Rd, Cambridge, CB2 1QP, UK. Correspondence and requests for materials should be addressed to A.I.A. (email: aabdi@kemri-wellcome.org) or P.C.B. (email: pb642@cam.ac.uk) 
been suggested to be as a result of a limitation in the diversity of important immune targets in P. falciparum ${ }^{30,31}$. Early serological work suggests the existence of PfEMP1 subgroups with limited diversity that are preferentially expressed by parasites causing infections in children with low immunity ${ }^{31-34}$.

Various studies have attempted to profile the var genes expressed by clinical $P$. falciparum isolates from children diagnosed with severe and mild malaria ${ }^{35-41}$. However, different results have been obtained from these studies. This is potentially due to differences in the methods used to measure var expression. Previously, we used an expressed sequence tag (EST) approach using DBL $\alpha$-tag amplification and sequencing to determine the var expression profile of clinical isolates ${ }^{38}$. With this method we found that the proportion of "group A-like" var genes expressed by the infecting parasites was positively associated with severe malaria and negatively associated with host antibodies present at the time of infection ${ }^{38,42}$. This supports the existence of PfEMP1 subsets with limited diversity $^{30-32}$. This result was consistent with other studies employing a similar EST approach ${ }^{36,43}$.

Other investigators have used real-time qPCR primers designed to quantify more directly the transcript abundance of var genes belonging to group A, B, and C or specific domain cassettes, relative to the expression of two metabolic genes $35,37,40,41$. These studies found that severe malaria is associated with the transcript quantity of group A, B and subsets of group A and B var genes containing domain cassette 13 and $8^{35,37,40,41}$. In one study, the transcript quantity of group C var genes was associated with severe malaria, particularly cerebral malaria ${ }^{39}$. Overall, the qPCR approach suggests that severe malaria is associated with the expression of multiple PfEMP1 subsets.

Two parasite encoded histone deacetylases commonly known as $P f s i r 2 a$ and $P f$ sir $2 b$ have been linked to differential regulation of var gene expression ${ }^{44,45}$. In a recent study on clinical isolates, expression levels of Pfsir2a and Pfsir2b were associated with what was described as a "dysregulation" of var gene expression ${ }^{41}$.

Since these various studies were conducted in different laboratories and on samples from different geographical areas, it is unclear whether parasites vary considerably between populations or whether the EST and qPCR approaches are providing different kinds of information.

To help resolve apparently conflicting results obtained from different studies, we brought together qPCR and EST approaches within a single dataset. We also analysed the expression of Pfsir2a, Pfsir2b together with two markers of gametocyte commitment, Pfap $2-\mathrm{g}^{46}$ and Pfs $16^{47}$.

We discuss how, despite apparent discrepancies seen previously between qPCR and EST, these approaches are highly consistent with one another but provide different kinds of information. We show that both global expression of var genes and expression of PfSir2a by the infecting parasite population is negatively associated with the breadth of host antibodies against the IE surface carried at the time of disease. We suggest that, in addition to switching between different PfEMP1 types, the parasite regulates the quantity of PfEMP1 on the IE globally as an additional immune evasion strategy.

\section{Results and Discussion}

To understand how PfEMP1 expression by the infecting parasite population is modified by host immunity at the time of infection, we compared var gene expression profiles and parasite IE phenotypes from parasites isolated from children with malaria attending Kilifi County Hospital in Kenya. We used three approaches: 1) an EST approach $^{37,38,40}$ that gives low resolution information about the proportion of expressed var sequence tags sampled from each isolate falling in broadly defined var classes, "cys2" and a subset of cys2 called "Group A-like" 48 . 2) We used qPCR to estimate what we will call the "transcript quantity" of each of several var gene classes relative to two housekeeping genes ${ }^{40}$ (primers used are listed in Table S1). 3) As an equivalent measure of the EST from this qPCR data, we obtained what we will call the "proportional expression" of each var gene subset by calculating expression of each var gene class as a proportion of the measured total detectable var transcript.

qPCR and EST approaches are consistent. The following observations demonstrated that qPCR and EST approaches to measuring var gene expression are consistent.

First, in line with previous studies ${ }^{42,49}$, both the proportional expression (gpA_prop) and the transcript quantity of group A var genes (gpA1 \& gpA2) were positively associated with rosetting (Fig. 1a and Fig. 1b respectively).

Second, proportional expression estimated through qPCR was highly correlated with equivalent proportions estimated by EST sequencing (Fig. 1c). Specifically, 1) proportional expression of group A var genes (gpA_prop) was positively correlated with group A-like expression measured using EST (Figs. 1c) and 2) proportional expression of group B and C var genes (b1_prop, c2_prop, and bc_prop) were negatively associated with group A-like expression measured using EST (Fig. 1c).

However, such consistency was not observed when qPCR estimates of transcript quantity of individual var classes were compared with proportional expression estimated through EST (Fig. 1d). Though transcript quantity of group A var genes was positively associated with group A-like expression estimated using EST, transcript quantity of group B and C var did not show expected negative associations with group A-like var or positive associations with cys4 (i.e. non-group A-like) var tags estimated using EST (Fig. 1d).

When the expression of different var subsets is calculated as a proportion of the total var transcript we implicitly make an assumption that individual parasites from different clinical isolates express equal total quantities of var transcript and simply make a choice in which var genes to express in a mutually exclusive manner. In this scenario, the difference in var expression patterns observed between isolates will be mainly due to differences in the proportion of parasites in the population expressing each var subset. If this had been the case, we would expect the transcript quantity of broad subsets of var within the bulk parasite population to be negatively correlated with one another. However, there was no evidence for such a relationship (see gpA1 vs b1 or c2, Fig. 2a). If expressions of different classes of var genes do not show clear negative associations with one another, this would 


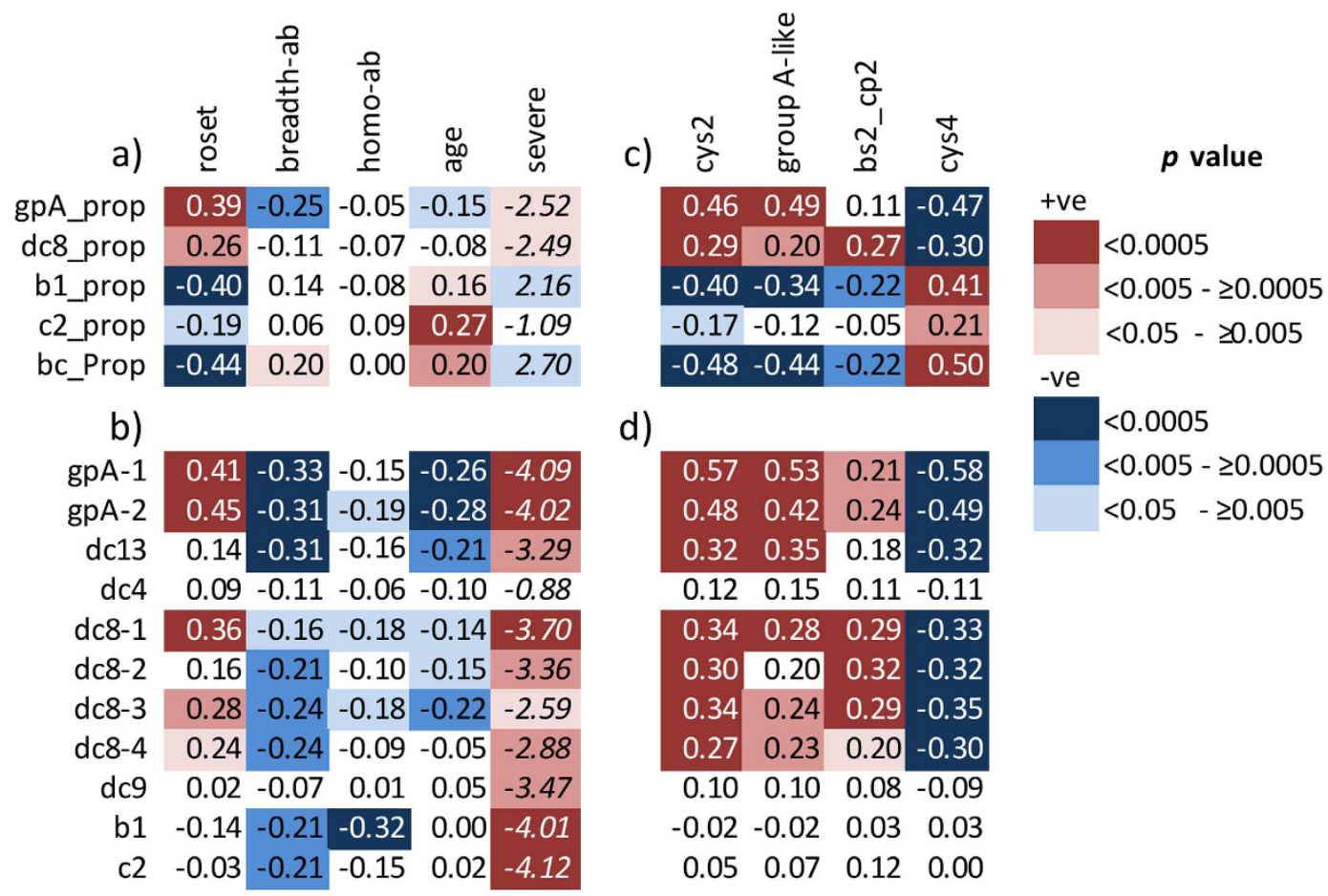

Figure 1. Comparisons of qPCR and EST methods of var expression analysis. Shown are Spearman's rank correlation coefficients (rho) or the Mann-Whitney U test's score (z). Mann-Whitney U test was used to test the difference between severe and non-severe malaria (negative $\mathrm{z}$ indicates positive association). Depth of the background shading represents the $\mathrm{p}$-value as indicated in the key. Red background indicates positive associations and blue background indicate negative associations. Increasing statistical significance is shown as deeper colour. (a) Associations between the "proportional expression" of broad classes of var calculated from qPCR and parasite surface phenotype; roset (rosetting), heterologous (breadth-ab) and homologous (hom-ab) antibodies present at the time of disease, age of the children (age) and severity of the disease (severe). breadth-ab = a measure of the breadth of reactivity of each child's antibodies present at the time of disease against infected erythrocytes of 8 heterologous $P$. falciparum clinical isolates, Homo- $\mathrm{Ab}=$ Antibodies against each child's own parasite isolate. gpA_prop, dc8_prop, b1_prop and c1_prop = "proportional expression" of group A, dc8, group B (b1) and C (c2) var genes respectively calculated from qPCR data. $\mathrm{bc} \_$prop $=$"proportional expression" of group B and C var genes together i.e bc_prop $=(\mathrm{b} 1+\mathrm{c} 2) / \mathrm{sum}$ var transcript (see method section). (b) The relationship between "transcript quantity" of var subclasses determined using qPCR with parasite surface phenotype (rosetting), heterologous and homologous antibodies present at the time of disease, age and disease severity (as for $(\mathbf{a})$ ). Names of the primers listed in Table S1 were used to represent the "transcript quantity" of the different var subclasses. (c) Associations between the "proportional expressions" of broad classes of var genes calculated from qPCR data to equivalent EST proportions calculated from expressed sequence tag (EST). bs2_cp2 is a subset of cys2 that may include group B var genes ${ }^{63}$.

(d) Associations between "transcript quantity" determined with the primers listed in Table S1 by qPCR and EST proportions. Group B (b1) and C (c2) transcript quantity did not show the expected negative association with group A-like or positive associations with cys4. $\mathrm{N}=191$ except for roset and homo-ab where $\mathrm{N}=121$.

suggest that the overall amount of var transcript in the parasite population can vary between isolates as has been suggested previously ${ }^{41}$.

Transcript quantity and proportional expression analyses differ because var gene expression varies globally. Relaxing the assumption of constant var expression levels between different isolates helps in the interpretation of the relationship between var expression and host immunity. Previous studies have shown that group A PfEMP1 are relatively conserved in comparison to non-group A PfEMP11-34,38. Consistent with this, proportional expression of group A var (gpA_prop) estimated by qPCR was negatively associated with IE antibodies (breadth-ab) carried at the time of disease (Fig. 1a, column 2), whilst proportional expression of groups $\mathrm{B}$ and $\mathrm{C}$ together (bc_prop) are positively associated with IE antibodies (Fig. 1a, column 2). However, when transcript quantity rather than proportional expression was considered, negative associations with IE antibodies extended to most major var gene subsets measured (Fig. 1b, column 2). This suggests that, rather than only selecting against parasites expressing one group of var genes over another, antibodies carried by the host may select more globally against high var gene expression.

This apparent global reduction of var gene expression with increasing host antibodies appears to be distinct from a reduction in the diversity of var genes that are expressed within each isolate. We previously used Simpsons diversity index to measure the "var expression homogeneity, $\mathrm{VEH}^{\text {" in these parasite isolates }}{ }^{50}$. The relationship 


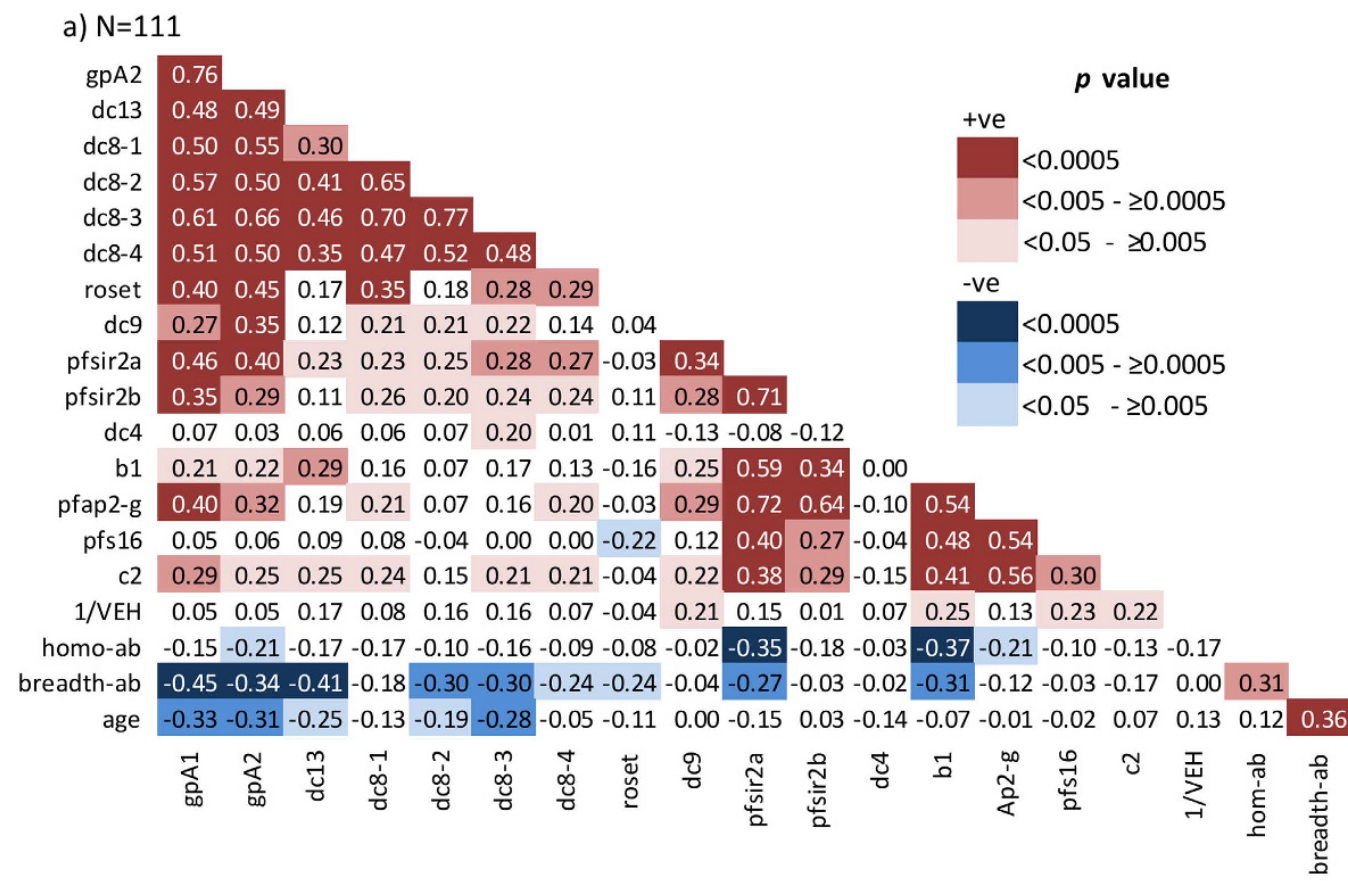

b) $\mathrm{N}=182$

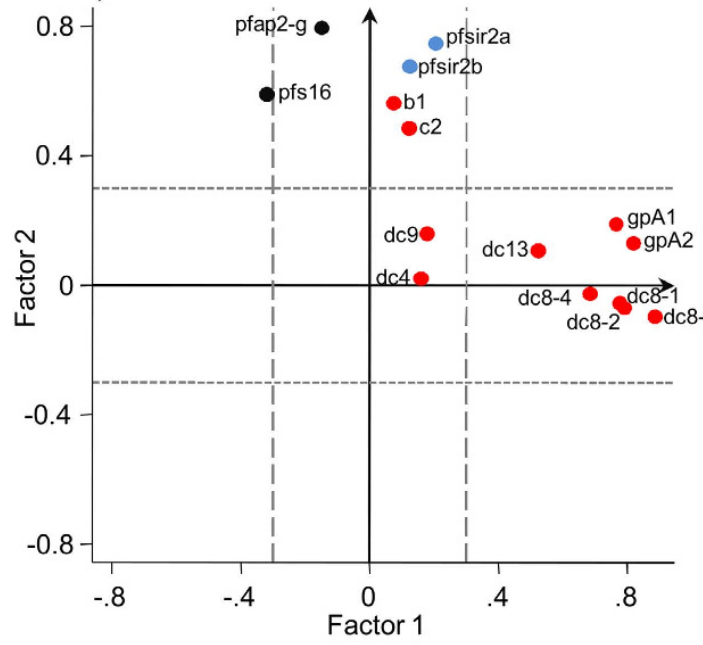

c)

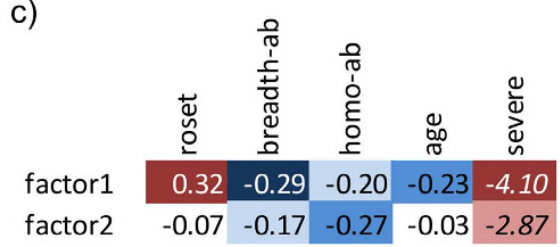

d) $\mathrm{N}=215$

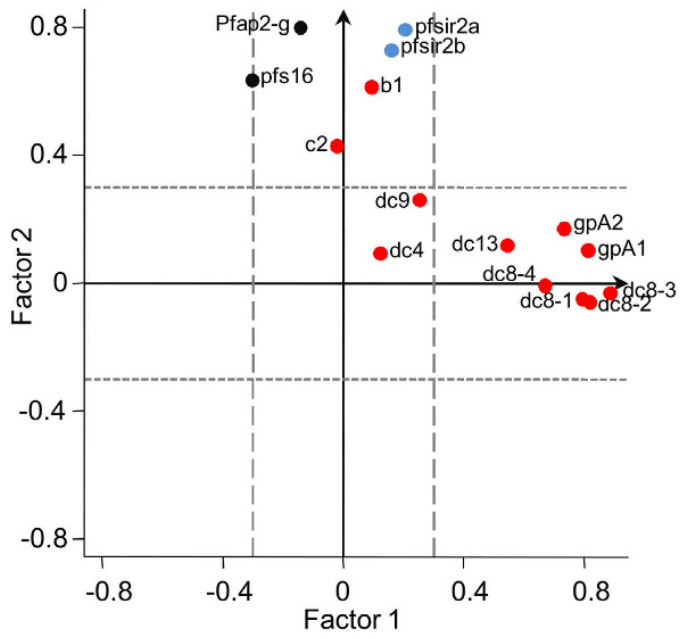

Figure 2. IE surface antibodies associated with global regulation of var gene expression. (a) Correlation matrix based on Spearman's rank correlation coefficient test. Shown is the rho value and the background shading was done as described in Fig. $1 . \mathrm{N}=111$. (b) Principal component factor analysis of var expression (clinical cases only). Red; transcript quantity of the var subclasses represented using the names of the primers (Table S1), Light blue; PfSIR2 expression (Pfsir2a, Pfsir2b), Black; markers of gametocytogenesis (Pfap-2g, Pfs16). Factor loadings above or below 0.3 were considered significant (dashed lines). $\mathrm{N}=182$ (c) The relationship between predicted factor scores of factor 1 and factor 2 with rosetting, breadth of host antibodies, host age, and severity of the disease $(\mathrm{N}=182$ except for roset and homo-ab where $\mathrm{N}=121)$. Shown is rho and $\mathrm{z}$ score derived from Spearman's rank correlation and Mann-Whitney U tests respectively. Background colouring was done in accordance with the p-value as described in Figs 1 and 2 legends. (d) Same as Fig. 2b except $\mathrm{N}=215$ and includes data of parasites from 33 children with asymptomatic infection. 
a)

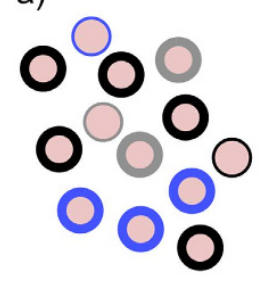

b)

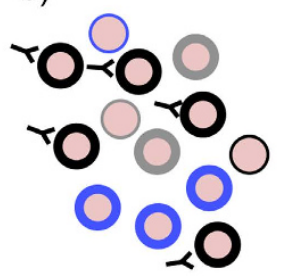

d)

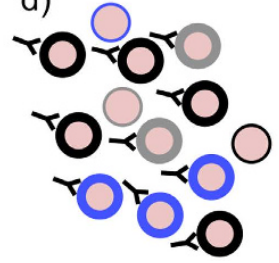

c)

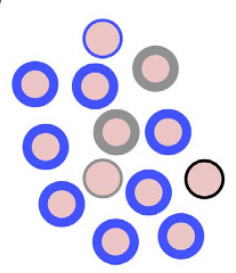

e)

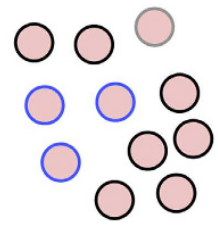

Figure 3. A hypothetical model for immunity dependent regulation of PfEMP1 expression. The black, blue and grey circles represent PfEMP1 variants expressed on the surface of the infected erythrocytes. $(\mathbf{a}-\mathbf{c})$ represents the response of parasites to partial immunity; (a) shows a hypothetical PfEMP1 expression profile within a naïve host where multiple PfEMP1 are expressed at high level to explore the host cytoadhesive space. Dominant variants are those which have a growth advantage due to their optimal cytoadhesion ability (b) antibody are raised first against the dominant variants (in this case the black type) (c) another variant (blue) previously expressed at lower frequency dominates the population. $(\mathbf{d}, \mathbf{e})$ represents the response of the parasite population in an individual carrying antibodies to a broad range of variants; (d) the pre-existing naturally acquired antibody clears all variants carrying high levels of PfEMP1, (e) A population of parasites expressing a previously recognised PfEMP1 but expressed at low levels of PfEMP1 survive and establish chronic infections.

between host antibodies and expressed var transcript quantity were independent of VEH in a regression analysis (Table S2).

We considered other possible causes of global variation in var gene expression. First, it is possible that varying commitment to gametocytogenes between parasite isolates may have led to global variation in var gene expression. Previous studies show that commitment to gametocytogenes is associated with an altered programme of expression of PfEMP $1^{51,52}$. Recent studies have further suggested that var expression is controlled globally by an epigenetic mechanism that is potentially linked to gametocytogenesis ${ }^{53,54}$. Second, previous studies have suggested a role for two genes Pfsir2a and Pfsir2b in the epigenetic control of var gene expression ${ }^{44,45}$. One recent study has suggested that epigenetic dysregulation of var genes may be associated with severe malaria ${ }^{41}$.

We therefore explored the expression of var in relation to two genes involved in the epigenetic regulation of var genes, Pfsir2a and Pfsir2 $\mathrm{b}^{41,44,45}$, two involved in early gametocyte differentiation, Pfap $2-\mathrm{g}^{46}$ and Pfs $16^{47}$, and host IE surface antibodies carried at the time of disease. Pfsir2a was positively associated with the var transcript quantity amplified with the primers gpA1, gpA2, dc8-2, dc8-2, dc8-3, dc9, b1 and c2, and markers of gametocyte commitment (Pfap2-g and Pfs16) (Fig. 2a).

IE antibodies carried at the time of disease (breadth-ab) were negatively associated with Pfsir2a expression ( $p=0.003$, Fig. 2a) but not with Pfsir2b, Pfap2-g or Pfs16 expression. Previously, higher body temperature was suggested to positively influence Pfsir2 expression ${ }^{41}$. We found that body temperature shows marginal positive associations with Pfsir2 expression (Pfsir2a; rho $=0.18, \mathrm{p}=0.05$, Pfsir $2 \mathrm{~b} ; \mathrm{rho}=0.18, \mathrm{p}=0.05, \mathrm{~N}=121$ ). However when body temperature and IE surface antibody was used as explanatory variables in a regression analysis predicting Pfsir2a, only IE surface antibodies showed independent association with Pfsir2a expression (Table S3). Taken together these data support the idea that antibodies may play a role in negatively selecting parasites in vivo according to their broadly defined epigenetic state and suggests that this is independent of gametocyte commitment ${ }^{55}$.

Principal factor analysis. To explore these relationships further we used principal factor analysis to visualize measures of var gene expression in relation to the expression of genes related to the control of var gene expression; Pfsir2a and Pfsir2b, and early markers of gametocyte differentiation; Pfap2-g and Pfs16.

Two major factors were identified in the dataset. The majority of the variables were significantly associated with one of these factors (Table S4). The different variables in the dataset were plotted in Fig. 3a in relation to each factor. Factor 1 accounts for approximately $65.84 \%$ of the variation in the data and correlates with expression of group A, DC8 and DC13 var genes (Fig. 2b and Table S4). Factor 2, which accounts for a further $28.18 \%$ of the variation in the data was associated with expression of group $\mathrm{B}$ and $\mathrm{C}$ var genes, epigenetic regulator genes; Pfsir2a and Pfsir2b, and markers of sexual commitment; Pfap2-g and Pfs16 (Fig. 2b and Table S4). Group B var genes, and Pfap2-g clustered with Pfsir2 and were associated with factor 2 (Table S4). This raises the possibility that expression of group B var genes and Pfap2-g may be linked together through a shared epigenetic mechanism as suggested by their clustering with Pfsir2.

To explore this further, we tested the associations of the predicted factor scores (see method) with rosetting, breadth of host antibodies present at time of disease, host age, and severity of the disease. As expected factor 1 
was associated with rosetting $(\mathrm{p}=0.0001$, Fig. $2 \mathrm{c})$, however, both factor 1 and 2 were negatively associated with breadth of recognition by host antibodies present at the time of disease and positively associated with severity of disease (Fig. 2c). This is consistent with the idea that antibodies carried by the host may select globally against high var gene expression.

Parasites that establish chronic infections need to survive in the presence of antibodies that have been accumulated prior to infection or developed during the course of the infection. In contrast, as a result of mosquito abundance, periods of high transmission lead to opportunities to infect naïve hosts or those that have not previously encountered that particular parasite genotype and carry low levels of antibody. The apparent global association of var gene expression with host antibodies described here may provide a simple external control for adaptation to these two essential phases in the parasite life cycle. To test this we extended this analysis to include var gene expression of parasites from asymptomatic individuals who carried infections towards the end of the low transmission season. The result of this extended data is consistent with that of the smaller dataset and clusters into two factors (Fig. 2d and Table S5). The predicted scores of both factors were negatively associated with breadth of host antibodies (factor 1 ; rho $=-0.4, \mathrm{p}<0.0001$, factor2; rho $=-0.33, \mathrm{p}<0.0001$, Spearman's correlation test) and asymptomatic infection (factor $1 ; \mathrm{z}=5.31, \mathrm{p}<0.0001$, factor $2 ; \mathrm{z}=6.7, \mathrm{p}<0.0001$, Mann-Whitney $\mathrm{U}$ test). This result raises the possibility that parasites causing asymptomatic infections under conditions of high levels of circulating antibodies may exhibit lower var gene expression (Fig. S1). This may be part of a mechanism to avoid immune surveillance and promote chronic infection. Despite the clustering of Pfap2-g and Pfs16 expression with factor 2 , there was no evidence for a direct correlation between IE antibody breadth and expression of Pfap 2-g (Fig. 2a). This does not support a direct modulation of gametocyte commitment by antibodies. It is none the less possible that our simple measure of the breadth of IE antibodies may not fully capture the immune selection pressure experienced by the parasite population at the time of infection.

To explore this further we considered "homologous" IE antibodies tested against cultured parasites from each child as a more direct measure of the immune pressure experienced by the infecting parasite population. There was a similar negative correlation between homologous IE antibodies and Pfsir2a and a marginal negative correlation between homologous IE antibodies and Pfap2-g expression (Fig. 2a). Determining whether antibody responses to the infecting parasite population play a direct role in selecting parasites that have reduced commitment to gametocytes will require a more extensive examination of the epigenetic state of cells that escape host antibodies.

In summary, the relatively independent expression of broad classes of var genes described here is consistent with a model (Fig. 3) that incorporates an extended parasite bet-hedging strategy in the face of host antibodies, in which var expression varies both in type and expression level. Such a strategy would potentially provide the parasite population with a simple means to explore the host cytoadhesive space by simultaneously expressing multiple PfEMP1 subsets at high quantities when infecting naïve hosts ${ }^{56}$. As part of a form of "balancing act" between 1) retaining the cytoadhesion function of IE and 2) immune evasion, expression of low quantities of PfEMP1 may also provide means for extending within-host parasite survival when host antibodies against the infecting isolates are high. This might explain why asymptomatic infection often express group A-like PfEMP $1^{50}$ but at low quantity (Fig.S1). This simple strategy driven by host antibodies may operate both among clinical infections of children and in the establishment of chronic infections. This model would potentially clarify the possible roles of other multigene families known to be expressed on the surface of IE and that undergo antigenic variation. Pfsir2a is also involved in the regulation of rifin genes expression ${ }^{45}$. In future experiments it would be interesting to determine whether expression of rifins and stevor antigens are altered in parasites that express lower levels of PfEMP1 on the surface.

\section{Material and Methods}

Clinical characteristics of the patients. Patient samples used in this study were described in previous publications ${ }^{38,42,50,57}$. The children presented to Kilifi County Hospital with severe and non-severe malaria. Severe malaria is defined as a hospital admission with impaired consciousness (Blantyre coma score $<4$ in patients under 8 months old; $<5$ in patients $\geq 8$ months old) ${ }^{58}$, respiratory distress (deep "kussmaul" pattern of breathing) $)^{59}$ or severe malarial anemia (hemoglobin $<5 \mathrm{~g} / \mathrm{dl}$ ). Non-severe cases include children admitted to hospital or attending out patient department who do not present with any of the three severe syndromes described above. Asymptomatic cases are children that were slides positive for $P$. falciparum but no clinical signs before the beginning of malaria season. The samples used in this study $(\mathrm{N}=215$, severe $=85$, non-severe $=97$, asymptomatic $=33$ ) are restricted to those for which 1) sufficient sample material remained for further study 2) we previously obtained var expression data using the dbl $\alpha$-tag or expressed sequenced tags (EST) analysis method and 3) a measure had been previously made of the breadth of IE surface antibodies response present at the time of disease ${ }^{38,42}$. These samples were collected between 2003-2007.

Ethics statement. Ethical approval for this study was obtained from Kenya Medical Research Institute (KEMRI) Ethical Review Committee (under SSC 1131), and written informed consent was obtained from parents/guardians of the study participants. The study methods were carried out in accordance with the approved guidelines.

RNA and cDNA preparations. RNA and cDNA preparation of the samples used in this study was described previously $^{38}$.

Rosetting and IE surface antibody. Rosette frequency and IE surface antibody data was obtained and published previously ${ }^{42}$. Rosetting data was described in ref. 42. IE surface antibody data was described in refs 
38,42 . Homologous antibody levels were also measured ${ }^{50}$ whereby each child's plasma was tested against that child's own infecting parasite matured in vitro to mid to late trophozoite stage. These data was previously published in ref. 50.

Var expression analysis. a) Expressed sequence tag (EST) sequencing approach. This data is previously published in ref. 38. Degenerate primers were used to PCR amplify a region within the DBL $\alpha$-domain that is highly conserved in the majority of var genes, cloned into TOPO ${ }^{\circledR}$-TA cloning vector, transformed into E. coli, and up to 96 colonies from each sample sequenced. The sequences were classified as described in ${ }^{38,42}$ and counted for each sample. EST proportional expression scores were obtained by counting the proportion of sequences within each sample that fell within each of the sequence groups. Group A-like tags were defined as those that had two cysteines (cys2) and carried one of a set of sequence blocks ${ }^{38}$.

b) Var transcript quantification using RT-PCR. Recently, 23 conserved domain cassettes (DCs) have been identified in PfEMP $1^{27}$. Primers targeting these domain cassettes were subsequently developed and used to quantify their expression in parasites from children with severe and non-severe malaria ${ }^{40}$. For the present study, we selected a subset of these primers that showed differential expression between severe and non-severe malaria. These included four primers targeting DC8 (named dc8-1, dc8-2, dc8-3, dc8-4), one primer for each of DC13 (dc13), DC4 (dc4), and DC9 (dc9), two primers targeting the majority of group A var genes (gpA1 and gpA2) (Table S1). We also quantified the expression of group B (b1) and C (c2) var genes using primers described in ${ }^{37}$ targeting the $5^{\prime}$-untranslated region of the var classes (Table S1). In addition we quantified the expression of two genes involved in epigenetic control of var gene expression, Pfsir2a and Pfsir2b, and two markers of early gametocytes commitment, Pfap2- $\mathrm{g}^{46}$ and $\mathrm{Pfs} 16^{47}$. We chose two housekeeping genes, Seryl tRNA synthetase and Fructose bisphosphate aldolase ${ }^{40,60,61}$ to use for relative quantification of the expressed var genes. We included dc9 targeting primer on the basis that dc9 was previously shown to be up-regulated in non-severe compared to severe malaria cases ${ }^{40}$. Before real-time PCR was carried out, the amplification efficiency of the primers was determined by generation of standard curves over 5 logs (100ng to 10pg of IT gDNA). The primers all had above $90 \%$ amplification efficiency. For the real time quantitative PCR, the PCR reaction and cycling conditions were carried out as described in ${ }^{40}$ with the Applied Biosystems 7500 Real-time PCR system. We set the cycle threshold (Ct) at 0.025. Controls with no template were included at the end of each batch of 22 samples per primer and the melt-curves analysed for non-specific amplification. We used genomic DNA from the IT4 laboratory parasite line at $10 \mathrm{ng} / \mu \mathrm{l}$ as a standard sample included in all plates because we were able to successfully amplify this line using all the primers used in this study. To estimate the "transcript quantity" the $\Delta \Delta$ ct relative quantification method was used to calculate the arbitrary transcript unit $\left(\mathrm{Tu}_{\mathrm{s}}\right)$ using the formula $\left(\mathrm{Tu}_{\mathrm{s}}=2^{(5-\Delta \Delta \mathrm{ct})}\right)$. However, we also estimated "proportional expression" of the transcripts within each sample (see below). When calculating proportional expression from qPCR the $\Delta \Delta \mathrm{ct}$ was not appropriate and we instead used $\mathrm{Tu}_{\mathrm{s}}$ calculated as described in Lavstsen et al. ${ }^{40}$ i.e. $2^{(5-\Delta c t)}$. We assigned a zero $\mathrm{Tu}_{\mathrm{s}}$ value if a reaction did not result in detectable amplification after 40 cycles of amplification, i.e if the Ct value was undetermined. We excluded from the analysis if amplification of either seryl tRNA synthetase or fructose biphosphate aldose could not be obtained.

Statistical analysis. To calculate the "proportional expression" of each var gene subset, we summed the transcript quantity of all the var subset analysed using the primers listed in table S1 (sum var transcript). The proportion contributed by each subset was then calculated. Since expression of group A and DC8 var subsets were each quantified using four different primers (Table S1), the median transcript quantity measured by these sets of group A and DC8 targeting primers was used to represent group A and DC8 respectively in the calculation of sum var transcript quantity and proportions. The sum of the transcript quantity of the var subsets was first obtained as follows; sum var transcript $=$ group A_median $+\mathrm{dc} 8 \_$median $+\mathrm{b} 1+\mathrm{c} 2$. The proportion of each subset was then calculated as follows; gpA_prop = group A_median/sum var transcript, dc8_prop=dc8_median/sum var transcript, b1_prop $=$ b1/sum var transcript, c2_prop $=\mathrm{c} 2 /$ sum $v a r$ transcript and bc_prop $=(\mathrm{b} 1+\mathrm{c} 2) / \mathrm{sum}$ var transcript. Correlations between variables were evaluated using Spearman's rank correlation coefficient or the 2-sample Wilcoxon rank-sum (Mann-Whitney) test.

Principal factor analysis. To visualize the relationships between the multiple variables and identify potential clusters of variables, we used principal factor analysis. We first checked whether our variables are factorable. We examined the factorability of the data using Kaiser-Meyer-Olkin (KMO) tests. KMO tests the adequacy of the data for factorial analysis and KMO above 0.6 is considered adequate ${ }^{62}$. To optimize the factor loadings (the relationship of each variable to the factors) and obtain simple structures where the variables are significantly associated with one of the factors and poorly on the others, the loadings were rotated using the promax rotation method and loading $>0.3$ or $<-0.3$ was considered significant. We used promax because it resulted in the best defined structure with the majority of variables loading above 0.3 or below -0.3 .

We used the Stata command "predict" to generate predicted factor scores for each individual. We then tested the relationship between rosetting, breadth of host antibodies, severity of the disease, asymptomatic infection and the factor scores using Spearman's rank correlation coefficient and Mann-Whitney U tests.

To eliminate zeros from the $\mathrm{Tu}_{\mathrm{s}}$ data we added 0.1 to all values and then log transformed the resultant $\mathrm{Tu}_{\mathrm{s}}$ values to normalize the distribution of the data before use in factor analysis. All statistical tests were performed using Stata software version 13 (Stata Corp, College Station, Tx).

\section{References}

1. Barnwell, J. W. et al. A human 88-kD membrane glycoprotein (CD36) functions in vitro as a receptor for a cytoadherence ligand on Plasmodium falciparum-infected erythrocytes. J Clin Invest 84, 765-772, doi: 10.1172/JCI114234 (1989). 
2. Oquendo, P., Hundt, E., Lawler, J. \& Seed, B. CD36 directly mediates cytoadherence of Plasmodium falciparum parasitized erythrocytes. Cell 58, 95-101, doi: 0092-8674(89)90406-6 (1989).

3. Berendt, A. R., Simmons, D. L., Tansey, J., Newbold, C. I. \& Marsh, K. Intercellular adhesion molecule-1 is an endothelial cell adhesion receptor for Plasmodium falciparum. Nature 341, 57-59, doi: 10.1038/341057a0 (1989).

4. Turner, L. et al. Severe malaria is associated with parasite binding to endothelial protein C receptor. Nature 498, 502-505, doi: 10.1038/nature12216 (2013).

5. Rowe, J. A., Moulds, J. M., Newbold, C. I. \& Miller, L. H. P. falciparum rosetting mediated by a parasite-variant erythrocyte membrane protein and complement-receptor 1. Nature 388, 292-295, doi: 10.1038/40888 (1997).

6. Rowe, J. A. et al. Mapping of the region of complement receptor (CR) 1 required for Plasmodium falciparum rosetting and demonstration of the importance of CR1 in rosetting in field isolates. J Immunol 165, 6341-6346 (2000).

7. Udomsangpetch, R., Todd, J., Carlson, J. \& Greenwood, B. M. The effects of hemoglobin genotype and ABO blood group on the formation of rosettes by Plasmodium falciparum-infected red blood cells. Am J Trop Med Hyg 48, 149-153 (1993).

8. Barragan, A., Kremsner, P. G., Wahlgren, M. \& Carlson, J. Blood group A antigen is a coreceptor in Plasmodium falciparum rosetting. Infect Immun 68, 2971-2975 (2000).

9. Kyes, S., Horrocks, P. \& Newbold, C. Antigenic variation at the infected red cell surface in malaria. Annu Rev Microbiol 55, 673-707, doi: 10.1146/annurev.micro.55.1.673 (2001)

10. Hanson, J. et al. Relative contributions of macrovascular and microvascular dysfunction to disease severity in falciparum malaria. $J$ Infect Dis. doi: 10.1093/infdis/jis400 (2012).

11. Su, X. Z. et al. The large diverse gene family var encodes proteins involved in cytoadherence and antigenic variation of Plasmodium falciparum-infected erythrocytes. Cell 82, 89-100, doi: 0092-8674(95)90055-1 (1995).

12. Baruch, D. I. et al. Cloning the P. falciparum gene encoding PfEMP1, a malarial variant antigen and adherence receptor on the surface of parasitized human erythrocytes. Cell 82, 77-87 (1995).

13. Smith, J. D. et al. Switches in expression of Plasmodium falciparum var genes correlate with changes in antigenic and cytoadherent phenotypes of infected erythrocytes. Cell 82, 101-110 (1995).

14. Gardner, M. J. et al. Genome sequence of the human malaria parasite Plasmodium falciparum. Nature 419, 498-511, doi: 10.1038/ nature01097 (2002)

15. Scherf, A. et al. Antigenic variation in malaria: in situ switching, relaxed and mutually exclusive transcription of var genes during intra-erythrocytic development in Plasmodium falciparum. EMBO J 17, 5418-5426, doi: 10.1093/emboj/17.18.5418 (1998).

16. Scherf, A., Lopez-Rubio, J. J. \& Riviere, L. Antigenic variation in Plasmodium falciparum. Annu Rev Microbiol 62, 445-470, doi: 10.1146/annurev.micro.61.080706.093134 (2008).

17. Bull, P. C. et al. Parasite antigens on the infected red cell surface are targets for naturally acquired immunity to malaria. Nat Med 4, 358-360 (1998).

18. Giha, H. A. et al. Antibodies to variable Plasmodium falciparum-infected erythrocyte surface antigens are associated with protection from novel malaria infections. Immunol Lett 71, 117-126 (2000).

19. Deitsch, K. W., del Pinal, A. \& Wellems, T. E. Intra-cluster recombination and var transcription switches in the antigenic variation of Plasmodium falciparum. Mol Biochem Parasitol 101, 107-116 (1999).

20. Freitas-Junior, L. H. et al. Frequent ectopic recombination of virulence factor genes in telomeric chromosome clusters of $\mathrm{P}$. falciparum. Nature 407, 1018-1022, doi: 10.1038/35039531 (2000).

21. Frank, M. et al. Frequent recombination events generate diversity within the multi-copy variant antigen gene families of Plasmodium falciparum. Int J Parasitol 38, 1099-1109, doi: 10.1016/j.ijpara.2008.01.010 (2008).

22. Duffy, M. F., Byrne, T. J., Carret, C., Ivens, A. \& Brown, G. V. Ectopic recombination of a malaria var gene during mitosis associated with an altered var switch rate. J Mol Biol 389, 453-469, doi: 10.1016/j.jmb.2009.04.032 (2009).

23. Taylor, H. M., Kyes, S. A. \& Newbold, C. I. Var gene diversity in Plasmodium falciparum is generated by frequent recombination events. Mol Biochem Parasitol 110, 391-397 (2000).

24. Claessens, A. et al. Generation of antigenic diversity in Plasmodium falciparum by structured rearrangement of Var genes during mitosis. PLoS genetics 10, e1004812, doi: 10.1371/journal.pgen.1004812 (2014).

25. Kraemer, S. M. et al. Patterns of gene recombination shape var gene repertoires in Plasmodium falciparum: comparisons of geographically diverse isolates. BMC Genomics 8, 45, doi: 10.1186/1471-2164-8-45 (2007).

26. Buckee, C. O. \& Recker, M. Evolution of the multi-domain structures of virulence genes in the human malaria parasite, Plasmodium falciparum. PLoS Comput Biol 8, e1002451, doi: 10.1371/journal.pcbi.1002451(2012).

27. Rask, T. S., Hansen, D. A., Theander, T. G., Gorm Pedersen, A. \& Lavstsen, T. Plasmodium falciparum erythrocyte membrane protein 1 diversity in seven genomes-divide and conquer. PLoS Comput Biol 6, doi: 10.1371/journal.pcbi.1000933 (2010).

28. Langhorne, J., Ndungu, F. M., Sponaas, A. M. \& Marsh, K. Immunity to malaria: more questions than answers. Nat Immunol 9, 725-732, doi: 10.1038/ni.f.205 (2008).

29. Goncalves, B. P. et al. Parasite burden and severity of malaria in Tanzanian children. N Engl J Med 370, 1799-1808, doi: 10.1056/ NEJMoa1303944 (2014).

30. Bull, P. C., Lowe, B. S., Kortok, M. \& Marsh, K. Antibody recognition of Plasmodium falciparum erythrocyte surface antigens in Kenya: evidence for rare and prevalent variants. Infect Immun 67, 733-739 (1999).

31. Nielsen, M. A. et al. Plasmodium falciparum variant surface antigen expression varies between isolates causing severe and nonsevere malaria and is modified by acquired immunity. J Immunol 168, 3444-3450 (2002).

32. Bull, P. C. et al. Plasmodium falciparum-infected erythrocytes: agglutination by diverse Kenyan plasma is associated with severe disease and young host age. J Infect Dis 182, 252-259, doi: 10.1086/315652 (2000).

33. Staalsoe, T. et al. In vitro selection of Plasmodium falciparum 3D7 for expression of variant surface antigens associated with severe malaria in African children. Parasite Immunol 25, 421-427, doi: 652 (2003).

34. Jensen, A. T. et al. Plasmodium falciparum associated with severe childhood malaria preferentially expresses PfEMP1 encoded by group A var genes. J Exp Med 199, 1179-1190, doi: 10.1084/jem.20040274 (2004).

35. Kaestli, M. et al. Virulence of malaria is associated with differential expression of Plasmodium falciparum var gene subgroups in a case-control study. J Infect Dis 193, 1567-1574, doi: 10.1086/503776 (2006).

36. Kyriacou, H. M. et al. Differential var gene transcription in Plasmodium falciparum isolates from patients with cerebral malaria compared to hyperparasitaemia. Mol Biochem Parasitol 150, 211-218, doi: 10.1016/j.molbiopara.2006.08.005 (2006)

37. Rottmann, M. et al. Differential expression of var gene groups is associated with morbidity caused by Plasmodium falciparum infection in Tanzanian children. Infect Immun 74, 3904-3911, doi: 10.1128/IAI.02073-05 (2006).

38. Warimwe, G. M. et al. Plasmodium falciparum var gene expression is modified by host immunity. Proc Natl Acad Sci USA 106, 21801-21806, doi: 10.1073/pnas.0907590106 (2009).

39. Kalmbach, Y. et al. Differential var gene expression in children with malaria and antidromic effects on host gene expression. J Infect Dis 202, 313-317, doi: 10.1086/653586 (2010).

40. Lavstsen, T. et al. Plasmodium falciparum erythrocyte membrane protein 1 domain cassettes 8 and 13 are associated with severe malaria in children. Proc Natl Acad Sci USA 109, E1791-1800, doi: 10.1073/pnas.1120455109 (2012).

41. Merrick, C. J. et al. Epigenetic dysregulation of virulence gene expression in severe Plasmodium falciparum malaria. J Infect Dis 205, 1593-1600, doi: 10.1093/infdis/jis239 (2012). 
42. Warimwe, G. M. et al. Prognostic indicators of life-threatening malaria are associated with distinct parasite variant antigen profiles. Sci Transl Med 4, 129ra145, doi: 10.1126/scitranslmed.3003247 (2012).

43. Kirchgatter, K. \& Portillo Hdel, A. Association of severe noncerebral Plasmodium falciparum malaria in Brazil with expressed PfEMP1 DBL1 alpha sequences lacking cysteine residues. Mol Med 8, 16-23, doi: S1528365802100164 (2002).

44. Duraisingh, M. T. et al. Heterochromatin silencing and locus repositioning linked to regulation of virulence genes in Plasmodium falciparum. Cell 121, 13-24, doi: 10.1016/j.cell.2005.01.036 (2005).

45. Tonkin, C. J. et al. Sir2 paralogues cooperate to regulate virulence genes and antigenic variation in Plasmodium falciparum. PLoS Biol 7, e84, doi: 10.1371/journal.pbio.1000084 (2009).

46. Kafsack, B. F. et al. A transcriptional switch underlies commitment to sexual development in malaria parasites. Nature 507, 248-252, doi: 10.1038/nature12920 (2014).

47. Dechering, K. J., Thompson, J., Dodemont, H. J., Eling, W. \& Konings, R. N. Developmentally regulated expression of pfs16, a marker for sexual differentiation of the human malaria parasite Plasmodium falciparum. Mol Biochem Parasitol 89, 235-244, doi: S0166-6851(97)00123-0 (1997).

48. Bull, P. C. et al. Plasmodium falciparum variant surface antigen expression patterns during malaria. PLoS Pathog 1, e26, doi: 10.1371/journal.ppat.0010026 (2005).

49. Vigan-Womas, I. et al. An in vivo and in vitro model of Plasmodium falciparum rosetting and autoagglutination mediated by varO, a group A var gene encoding a frequent serotype. Infect Immun 76, 5565-5580, doi: 10.1128/IAI.00901-08 (2008).

50. Warimwe, G. M. et al. Plasmodium falciparum var Gene Expression Homogeneity as a Marker of the Host-Parasite Relationship under Different Levels of Naturally Acquired Immunity to Malaria. PLoS One 8, e70467, doi: 10.1371/journal.pone.0070467 (2013).

51. Tiburcio, M. et al. Early gametocytes of the malaria parasite Plasmodium falciparum specifically remodel the adhesive properties of infected erythrocyte surface. Cell Microbiol 15, 647-659, doi: 10.1111/cmi.12062 (2013)

52. Tiburcio, M., Sauerwein, R., Lavazec, C. \& Alano, P. Erythrocyte remodeling by Plasmodium falciparum gametocytes in the human host interplay. Trends Parasitol 31, 270-278, doi: 10.1016/j.pt.2015.02.006 (2015).

53. Brancucci, N. M. et al. Heterochromatin protein 1 secures survival and transmission of malaria parasites. Cell Host Microbe 16, 165-176, doi: 10.1016/j.chom.2014.07.004 (2014).

54. Coleman, B. I. et al. A Plasmodium falciparum histone deacetylase regulates antigenic variation and gametocyte conversion. Cell Host Microbe 16, 177-186, doi: 10.1016/j.chom.2014.06.014 (2014).

55. Rovira-Graells, N. et al. Transcriptional variation in the malaria parasite Plasmodium falciparum. Genome research 22, 925-938, doi: 10.1101/gr.129692.111 (2012)

56. Wang, C. W. et al. The Plasmodium falciparum var gene transcription strategy at the onset of blood stage infection in a human volunteer. Parasitol Int 58, 478-480, doi: 10.1016/j.parint.2009.07.004 (2009).

57. Abdi, A. I. et al. Plasmodium falciparum antigenic variation: relationships between widespread endothelial activation, parasite PfEMP1 expression and severe malaria. BMC Infect Dis 14, 170, doi: 10.1186/1471-2334-14-170 (2014).

58. Berkley, J. A., Mwangi, I., Mellington, F., Mwarumba, S. \& Marsh, K. Cerebral malaria versus bacterial meningitis in children with impaired consciousness. QJM 92, 151-157 (1999).

59. English, M. et al. Deep breathing in children with severe malaria: indicator of metabolic acidosis and poor outcome. Am J Trop Med Hyg 55, 521-524 (1996)

60. Salanti, A. et al. Evidence for the involvement of VAR2CSA in pregnancy-associated malaria. J Exp Med 200, 1197-1203, doi: 10.1084 /jem.20041579 (2004)

61. Salanti, A. et al. Selective upregulation of a single distinctly structured var gene in chondroitin sulphate A-adhering Plasmodium falciparum involved in pregnancy-associated malaria. Mol Microbiol 49, 179-191 (2003).

62. HF, K. An index of factor simplicity. Psychometrika 39, 31-36 (1974).

63. Bull, P. C. et al. Plasmodium falciparum antigenic variation. Mapping mosaic var gene sequences onto a network of shared, highly polymorphic sequence blocks. Mol Microbiol 68, 1519-1534, doi: 10.1111/j.1365-2958.2008.06248.x (2008).

\section{Acknowledgements}

We are grateful to the children and their parents/guardians for participation in the study. We thank Jennifer Musyoki and Michael Opiyo for technical assistance. This work was supported by Wellcome Trust Programme grants (084535 and 092741). A.A. was also supported by Wellcome Trust Training grant in Public Health and Tropical Medicine (103956) and a Wellcome Trust strategic award (084538). This paper is published with the permission of the director of KEMRI.

\section{Author Contributions}

A.A., G.W., C.K., E.K. and M.M. generated the var expression data, G.F. helped in the statistical analysis, A.A. and P.B. wrote the manuscript, and P.B. provided the overall supervision of the work. All authors approved the final version of the manuscript.

\section{Additional Information}

Supplementary information accompanies this paper at http://www.nature.com/srep

Competing financial interests: The authors declare no competing financial interests.

How to cite this article: Abdi, A. I. et al. Global selection of Plasmodium falciparum virulence antigen expression by host antibodies. Sci. Rep. 6, 19882; doi: 10.1038/srep19882 (2016).

(c) (i) This work is licensed under a Creative Commons Attribution 4.0 International License. The images or other third party material in this article are included in the article's Creative Commons license, unless indicated otherwise in the credit line; if the material is not included under the Creative Commons license, users will need to obtain permission from the license holder to reproduce the material. To view a copy of this license, visit http://creativecommons.org/licenses/by/4.0/ 